\title{
Effects of the six-minute walking test on dyspnea and activities of daily living in pneumoconiosis patients
}

\author{
Eun-Jin Kim', Hye-Soon Kim²,* Myung-Hee Lee ${ }^{3}$ \\ 'Department of Nursing, Medical Station, Ansan Workers' Compensation Hospital, Ansan, Korea \\ ${ }^{2}$ Department of Nursing, Gachon University, Incheon, Korea \\ ${ }^{3}$ Department of Nursing, Shin Sung University, Dangjin-gun, Chungnam, Korea
}

The purpose of this study was to examine the effects of walking exercise through a 6-min walking test on subjective dyspnea, pulmonary function test, arterial blood gas analysis, and activities of daily living to provide base data for the nursing intervention of pneumoconiosis patients. The subjects were pneumoconiosis patients men aged over 60 . This quasi-experimental study was designed with a non-equivalent control group pre- and post-test design, and the study period was September to December 2013. Of the pneumoconiosis inpatient subjects, 39 were assigned to the experimental group and the 39 to the control group. Only the experimental group was subjected to a 6 -min walking test three times a week for 10 weeks. Subjective dyspnea was measured based on anxiety about dyspnea during and after the exercise. FEV1\% prediction and FEV1/FVC\% were used to measure the pulmo-

\section{INTRODUCTION}

Pneumoconiosis is a chronic pulmonary disease caused by the accumulation of small (0.5- to 5-um) dusts in the lungs, which leads to inflammation and fibrosis in the air sacs. The function of the lungs worsens with the disease progress, and the patients show dyspnea, coughing, sputum, and chest pains. In particular, coal workers' pneumoconiosis has a higher risk of complications than normal pneumoconiosis, and the patients have dyspnea when the symptoms are severe. Its onset date is about $10 \mathrm{yr}$ after the first exposure to coal dust, and as such, more than $50 \%$ of the patients are over $60 \mathrm{yr}$ old (Korean Occupational Safety and Health Agency, 2011).

Pneumoconiosis is an irreversible disease with no cure. The decreased activities due to decreased breathing function weaken the respiratory muscles and worsen dyspnea (Bauer et al., 2007; Sir- nary function. The arterial blood gas was measured based on the $\mathrm{pH}$, $\mathrm{PaO}_{2}, \mathrm{PaCO}_{2}$, and activities of daily living. The data were analyzed through an $\chi^{2}$ and $t$-test. The study results showed that the 6 -min walking test reduced the anxiety about dyspnea of the pneumoconiosis patients during the exercise, moderated the dyspnea index after the performance of the activities, and improved the patients' ability to perform activities of daily living. Thus, walking exercise seems to be an effective nursing intervention to maintain the respiratory rehabilitation outcomes by minimizing the pneumoconiosis patients' burden.

Keywords: Walking, pneumoconiosis, dyspnea, Pulmonary function test, Activities of daily living
${ }^{*}$ Corresponding author: Hye-Soon Kim

Department of Nursing, Gachon University (Medical Campus),

191 Hambakmoero, Yeonsu-gu, Incheon 406-799, Korea

Tel: +82-32-820-4208, Fax: +82-32-820-4201, E-mail: hsoon587@yahoo.co.kr

Received: June 30, 2014 / Accepted: September 30, 2014 ajuddin and Kanne, 2009).The patients feel anxious about experiencing shortage of breath while engaging in physical activities, which limits their daily activities (Leidy and Haase, 1996).

The rehabilitation program for patients with respiratory disease aims to decrease the symptoms, increase the patients' participation in daily as well as mental and social activities, and to eventually improve the quality of life of patients with chronic respiratory disease (Kim and Kang, 2006). In this study, the previous studies on respiratory rehabilitation programs (Han, 2003; Kim and Kang, 2006; No et al., 2009) were reviewed. Various rehabilitation programs developed from many studies have been applied, but a standardized exercise program for chronic lung disease patients has yet to be established. The most important part of respiratory rehabilitation is aerobic exercise (Kim, 2003), and the typical aerobic exercise is walking. Walking is a systemic exercise, and 
most daily activities involve walking. As such, it is considered a suitable exercise for elderly people.

The American Thoracic Society (ATS, 1995) reported that the ultimate goal of the nursing and treatment of chronic lung disease is to help patients actively lead the management of their own health to prevent complications, minimize symptoms, and maintain their body functions at the optimal levels for carrying out daily activities under the given circumstances.

The six-minute walking test is suggested as a useful test for evaluating the treatment efficacy by assessing the pulmonary function and exercise ability of patients with pulmonary disease (ATS, 2002). The test involves the measurement of the walking distance covered by a patient for a certain period of time, with an adjustable walking speed. As the test involves a similar amount of exercise during daily activities, it can directly reflect the lung functions and exercise abilities of patients while carrying out daily activities. It does not require special equipment and is simple, safe, and has high reproducibility (Stark et al., 1982). The six-minute walking test was reported to have higher sensitivity in measuring the symptoms of dyspnea in chronic obstructive pulmonary disease (COPD) than the lung function test or arterial blood gas analysis (Lee et al., 1992).

Based on the investigators' clinical experience in the pulmonology department, it was found that the patients with chronic pulmonary diseases such as pneumoconiosis feel financially burdened by the respiratory rehabilitation program due to their long-term hospitalization for treatment, and also have difficulty applying the respiratory rehabilitation and exercise program to their daily activities. As the respiratory rehabilitation and exercise program for chronic pulmonary disease patients needs to be applied for a long time for long-term efficacy, the patients should be able to easily perform the exercise without assistance from trained staff or the use of special equipment in a comfortable environment.

This study was conducted to prove the efficacy of the six-minute walking exercise on dyspnea and the ability to perform daily activities through a six-minute walking test that subjectively evaluates dyspnea and exercise ability with self-adjusting exercise intensity based on the patient's physical ability. Also, it aimed to investigate the nursing intervention for patients with pneumoconiosisthrough walking exercise by motivating patients to exercise and by improving their confidence.

\section{MATERIALS AND METHODS}

\section{Setting and sample participants}

The design of this study was a time series with a non-equiva- lent control group quasi-experimental design to analyze the efficacy of six-minute walking on dyspnea and daily activity performance in patients with pneumoconiosis.

The study subjects were over-60-year-old male pneumoconiosis W Hospital (in Gyeonggi-do) inpatients from September 30 to December 6, 2013. The detailed inclusion/exclusion criteria are as follows: patients who understood the purpose of this study and who agreed to participate in it; with pneumoconiosis and were considered eligible to perform the program based on the investigator's judgment; whose medications for pneumoconiosis or hypertension had not changed for the last one month; without severe heart disease, cerebrovascular disease, cancer, orthopedic, or neuropsychological disease; and who had not done regular exercise for the last one month.

The sample size of this study was determined to be 39 subjects for each group based on the G*Power 3.0 program (Faul et al., 2007), with a 0.5 effect size, 0.7 statistical power, and 0.05 significance level. Forty subjects were originally enrolled for each group ( 40 for the experimental group and 40 for the control group, for a total of 80), but one subject in the experimental group was withdrawn from the study due to hospital discharge, and one subject in the control group was withdrawn due to symptom aggravation. As such, 39 subjects in each group were able to complete the study. To prevent the subjects from each group from sharing information with those in the other group, the subjects were hospitalized in different wards, and the subjects in the experimental group were given an explanation about the study results.

\section{Instrumentation}

A six-minute walking exercise is an exercise using a six-minute walking test. The six-minute walking test is described in the ATS guideline (2002) as involving walking on 30-meter hospital floors where every 3 meters are marked. The patients are made to wear comfortable clothes and shoes during the test. The walking is selfpaced, and the patients are allowed to take a break as required. The subjects wait at the starting line and are asked to walk the 30-meter distance back and forth at their fastest speed, starting with the signal. For the subjective evaluation of dyspnea in this study, the anxiety about dyspnea during the exercise was scored on a scale of 1 (no anxiety) to 5 (very severe anxiety). The higher the score was, the greater the anxiety about dyspnea during the exercise. The degree of dyspnea after the exercise was scored on a scale of 0 (no shortness of breath) to 10 (severe shortness of breath) using a 12-level modified Borg scale (Borg, 1982). The shortness of breath for the last two weeks was evaluated by the subject using 
the Borg scale dyspnea index, and the higher score was, the more severe the dyspnea. The pulmonary function, which involves pulmonary expansion, was used in this study as an objective indicator of the disease severity as a volume/time relationship using the pulmonary volume, functional capacity, and resistance in the trachea. A trained examiner used a pulmonary test device (Vmax$22+\mathrm{V} 62 J$, Sensormedics, USA) to measure the FEV1\% prediction (pred.) and FEV1/FVC\%, of the pulmonary function indicators. A more than $50 \%$ FEV1\% pred. was considered mild or stage I; 35$49 \%$, stage II; and $34 \%$ or less, stage III. For the activities of daily living (ADL), the modified chronic respiratory disease questionnaire (Guyatt et al., 1987) formulated by Han (2003), consisting of 23 questions, was used for this study. Each question in the questionnaire was scored on a scale of 1 (cannot be performed) to 5 (not difficult to perform). The lowest score was 23 points, and the highest, 115 points. The higher the score was, the more the subject could independently perform the daily activity.

\section{Survey procedures and data processing}

Approval for this study was obtained from the institutional review board (IRB) of G University Hospital (GBIRB2013-161) and from the medical team, physicians, nursing department, and head nurse of the wards in W Hospital, and support for the study was obtained from the nurse team in the wards. Two nurses in the ward were trained about the purpose of the study method before the data collection and were involved in the data collection, along with the investigator. The investigator visited the patients' rooms in person to explain the study purpose and necessity as well as the confidentiality of the patients' information, and a signed informed-consent form was obtained from each subject. The data that were required to be collected before the test were obtained by the investigator a day before the walking test. The questionnaire was explained to the subjects, and the anxiety about the breathing difficulties that occurred during the exercise, the symptoms and severity of the dyspnea for the last two weeks, and the daily activities were evaluated by the subjects themselves. The experimental group was trained about the preparation exercise before the test, the six-minute walking exercise, and the finishing-up exercise. The subjects in the experimental group underwent pulse and blood pressure measurement before the walking exercise. If a subject showed any abnormal finding in the measurement, they were controlled before the exercise. To minimize the effect of the activities undertaken prior to the test, the subjects were asked to rest for more than 10 min before the test. For the preparation exercise, free gymnastics, stretching, and light walking were freely per- formed for five minutes, and six-minute walking exercise was likewise performed. For the finishing exercise, two sets of pursedlip breathing (10 times per set) were carried out to regulate the breathing, and then free gymnastics, stretching, and walking slowly were freely performed for five minutes. The preparation to the finishing-up exercises took about $20 \mathrm{~min}$. The experimental group was asked to perform the exercise series once a day (at around the same time) three times a week for 10 weeks. The distance of the six-minute walking exercise was recorded in the logbook. The post-investigation data were collected from the experimental and control groups 10 weeks after the initiation of the experiment. The experimental group was advised about the six-minute walking method and was informed of the study results after 10 weeks.

\section{Data analysis}

The study data were analyzed using the SPSS WIN 18.0 program. The general characteristics of the subjects were presented as frequency, percentage, mean, and standard deviation. The homogeneity of the general characteristics of the experimental and control groups was analyzed using a $\chi^{2}$-test and an independent t-test (Table 1), and the homogeneity of the subjective dyspnea (anxiety about breathing difficulties, MBS), ADL, and pulmonary function test (PFT) of the experimental and control groups was analyzed using an independent t-test (Table 2). The differences in subjective dyspnea, ADL, and PFT between the experimental and control groups were also analyzed using an independent t-test.

\section{RESULTS}

The difference in the change in the score of anxiety about breathing difficulties during exercise between the experimental group, which performed the six-minute walking exercise, and the control group, which did not perform the six-minute walking exercise, was analyzed. The score of the experimental group decreased from 2.89 to 2.69 while that of the control group increased from 2.92 to 3.15 , which were statistically significant differences $(P<0.05)$ (Table 3).

With regard to the difference in the change in the MBS between the experimental and control groups, the score of the experimental group decreased from 3.05 to 2.54 while that of the control group decreased from 2.87 to 2.82 , showing a statistically significant difference $(P<0.05)$ (Table 3$)$.

As for the difference in the change in the pulmonary function test results between the experimental and control groups, which- 
Table 1. Homogeneity test for the general characteristics

$(n=78)$

\begin{tabular}{|c|c|c|c|c|c|}
\hline \multirow{2}{*}{ Characteristics } & \multirow{2}{*}{ Division } & \multirow{2}{*}{$\begin{array}{c}\text { Exp. }(n=39) \\
n(\%) \text { or } M \pm S D\end{array}$} & \multirow{2}{*}{$\begin{array}{c}\text { Cont. }(\mathrm{n}=39) \\
\mathrm{n}(\%) \text { or } \mathrm{M} \pm \mathrm{SD}\end{array}$} & \multirow{2}{*}{$\chi^{2} / t$} & \multirow{2}{*}{$P$} \\
\hline & & & & & \\
\hline Age (yr) & & $70.74 \pm 5.4$ & $71.00 \pm 5.3$ & -0.21 & 0.834 \\
\hline Past history & $\begin{array}{l}\text { Hypertension } \\
\text { Diabetes mellitus } \\
\text { Tuberculosis } \\
\text { Hepatitis }\end{array}$ & $\begin{array}{r}12(30.8) \\
10(25.6) \\
5(12.8) \\
12(30.8)\end{array}$ & $\begin{array}{r}16(41.0) \\
7(17.9) \\
7(17.9) \\
9(23.1)\end{array}$ & 1.86 & 0.601 \\
\hline Smoking & $\begin{array}{l}\text { Yes } \\
\text { No } \\
\text { No answer }\end{array}$ & $\begin{array}{r}11(28.2) \\
24(61.5) \\
4(10.3)\end{array}$ & $\begin{array}{r}11(28.2) \\
19(48.7) \\
9(23.1)\end{array}$ & 2.50 & 0.286 \\
\hline Smoking period* (yr) & $\begin{array}{l}1-5 \\
6-10 \\
11-15 \\
16-20 \\
\geq 21 \\
\text { No answer }\end{array}$ & $\begin{array}{c}3(7.7) \\
3(7.7) \\
2(5.1) \\
7(17.9) \\
20(51.3) \\
4(10.3)\end{array}$ & $\begin{array}{c}1(2.6) \\
2(5.1) \\
3(7.7) \\
5(12.8) \\
19(48.7) \\
9(23.1)\end{array}$ & 3.68 & 0.596 \\
\hline Smoking amount (ea/day) & & $10.08 \pm 6.29$ & $9.38 \pm 7.03$ & 0.45 & 0.648 \\
\hline Regular exercise & $\begin{array}{l}\text { Yes } \\
\text { No }\end{array}$ & $\begin{array}{c}0(0.0) \\
39(100.0)\end{array}$ & $\begin{array}{c}0(0.0) \\
39(100.0)\end{array}$ & - & - \\
\hline $\begin{array}{l}\text { Low-dose oxygen therapy* } \\
\text { (time/day) }\end{array}$ & $\begin{array}{l}\text { Do not use } \\
1-2 \\
3-5 \\
\geq 6\end{array}$ & $\begin{array}{c}25(64.1) \\
2(5.1) \\
9(23.1) \\
3(7.7)\end{array}$ & $\begin{array}{c}22(56.4) \\
6(15.4) \\
9(23.1) \\
2(5.1)\end{array}$ & 3.14 & 0.162 \\
\hline ABGA & $\begin{array}{l}\mathrm{pH} \\
\mathrm{PaO}_{2}(\mathrm{mmHg}) \\
\mathrm{PaCO}_{2}(\mathrm{mmHg})\end{array}$ & $\begin{array}{c}7.40 \pm 0.02 \\
86.67 \pm 14.66 \\
39.35 \pm 2.25\end{array}$ & $\begin{array}{l}7.41 \pm 0.03 \\
90.01 \pm 12.29 \\
38.12 \pm 3.44\end{array}$ & $\begin{array}{r}-1.60 \\
-1.09 \\
1.87\end{array}$ & $\begin{array}{l}0.113 \\
0.279 \\
0.064\end{array}$ \\
\hline
\end{tabular}

*Fisher's exact test.

Exp., experimental group; Cont., control group; ABGA, arterial blood gas analysis.

Table 2. Homogeneity test for the variables related to sixminute walking

$(n=78)$

\begin{tabular}{llcccc}
\hline \multirow{2}{*}{ Variables } & & Exp. $(\mathrm{n}=39)$ & Cont. $(\mathrm{n}=39)$ & $\mathrm{t}$ & \\
\cline { 3 - 5 } & & $\mathrm{M} \pm \mathrm{SD}$ & $\mathrm{M} \pm \mathrm{SD}$ & & \\
\hline Subjective dyspnea & Anxiety about breathing difficulties during exercise (score) & $2.89 \pm 0.64$ & $2.92 \pm 0.76$ & & -0.17 \\
& Perception of dyspnea after exercise MBS (score) & $3.05 \pm 0.68$ & $2.87 \pm 0.52$ & 1.30 & 0.863 \\
PFT & FEV1 (\% pred.) & $65.79 \pm 24.13$ & $65.51 \pm 22.46$ & 0.05 & 0.198 \\
& FEV1/FVC (\%) & $81.33 \pm 20.72$ & $80.15 \pm 21.93$ & 0.24 & 0.958 \\
ADL (score) & & $61.46 \pm 5.40$ & $61.51 \pm 6.15$ & -0.21 & 0.808 \\
\hline
\end{tabular}

Exp., experimental group; Cont., control group; MBS, modified Borg scale; ADL, activities of daily living; PFT, pulmonary function test; FEV1 (\% pred.), forced expiratory volume in 1 second (\% predictive value): FEV1/FVC, forced expiratory volume in 1 second/forced vital capacity.

was analyzed using the forced expiratory volume for 1-second prediction (FEV1\% pred.), the forced vital capacity of prediction (FVC\% pred.), and the FEV1\% pred,, the ratio between FVC and FEV1, the FEV1\% pred. result showed an increase from 65.79 to $68.69 \%$ in the experimental group and from 65.51 to $65.77 \%$ in the control group, which were not statistically significantly differences ( $\mathrm{t}=0.05, P=0.958$ ) (Table 3 ). The ratio between the FVC and FEV1 (FVC/FEV1) results showed increases from 81.33 to
$83.77 \%$ in the experimental group and from 80.15 to $81.18 \%$ in the control group, with a statistically insignificant difference (Table 3).

For the difference in the change in ADL between the experimental and control groups, the analysis results showed increases from 61.46 to 64.49 in the experimental group and from 61.46 to 61.51 in the control group, showing a statistically significant difference $(P<0.05)$ (Table 3$)$. 
Table 3. Comparison of subjective dyspnea, ADL, and PFT between the groups

$(\mathrm{n}=78)$

\begin{tabular}{|c|c|c|c|c|c|c|c|}
\hline \multirow{2}{*}{ Variables } & & \multirow{2}{*}{ Groups } & Pretest & \multirow{2}{*}{$\begin{array}{c}\text { Post } 10 \text { weeks } \\
\mathrm{M} \pm \mathrm{SD}\end{array}$} & \multirow{2}{*}{$\begin{array}{c}\text { Differences } \\
\mathrm{M} \pm \mathrm{SD}\end{array}$} & \multirow{2}{*}{$\mathrm{t}$} & \multirow{2}{*}{$P$} \\
\hline & & & $\mathrm{M} \pm \mathrm{SD}$ & & & & \\
\hline \multirow[t]{4}{*}{ Subjective dyspnea } & Anxiety about breathing & Exp. $(n=23)$ & $2.89 \pm 0.64$ & $2.69 \pm 0.46$ & $0.20 \pm 0.52$ & -3.85 & 0.000 \\
\hline & difficulties during exercise (score) & Cont. $(n=23)$ & $2.92 \pm 0.76$ & $3.15 \pm 0.57$ & $-0.22 \pm 0.61$ & & \\
\hline & Perception of dyspnea after & Exp. $(n=23)$ & $3.05 \pm 0.68$ & $2.54 \pm 0.64$ & $0.51 \pm 0.60$ & -2.15 & 0.034 \\
\hline & exercise MBS (score) & Cont. $(n=23)$ & $2.87 \pm 0.52$ & $2.82 \pm 0.50$ & $0.05 \pm 0.55$ & & \\
\hline \multirow[t]{4}{*}{ PFT } & FEV1 (\% pred.) & Exp. $(n=23)$ & $65.79 \pm 24.13$ & $68.69 \pm 26.30$ & $-2.89 \pm 5.34$ & 0.05 & 0.958 \\
\hline & & Cont. $(n=23)$ & $65.51 \pm 22.46$ & $65.77 \pm 23.24$ & $-0.25 \pm 6.54$ & & \\
\hline & FEV1/FVC (\%) & Exp. $(n=23)$ & $81.33 \pm 20.72$ & $83.77 \pm 21.48$ & $-2.89 \pm 5.34$ & 0.56 & 0.574 \\
\hline & & Cont. $(n=23)$ & $80.15 \pm 21.93$ & $81.18 \pm 18.96$ & $-1.02 \pm 10.78$ & & \\
\hline \multirow{2}{*}{\multicolumn{2}{|c|}{ ADL (score) }} & Exp. $(n=23)$ & $61.46 \pm 5.40$ & $64.49 \pm 4.48$ & $-3.02 \pm 2.05$ & 2.44 & 0.017 \\
\hline & & Cont. $(n=23)$ & $61.51 \pm 6.15$ & $61.51 \pm 6.15$ & $0.23 \pm 1.28$ & & \\
\hline
\end{tabular}

Exp., experimental group; Cont., control group; MBS, modified Borg scale; ADL, activities of daily living; PFT, pulmonary function test; FEV1 (\% pred.), forced expiratory volume in 1 second (\% predictive value); FEV1/FVC, forced expiratory volume in 1 second/forced vital capacity.

\section{DISCUSSION}

The ultimate goal of the nursing and treatment of patients with chronic pulmonary disease is to help the patients actively manage their health to minimize the symptoms, prevent complications, and maintain their functions for daily activities under the given circumstances. The COPD patients in all stages can increase their functions for physical activities through the exercise program, can attain partial respiratory rehabilitation, and can relieve their symptoms, including dyspnea and fatigue, but a standardized program has yet to be established. As such, the efficacy of six-minute walking exercise on dyspnea and ADL were investigated in this study, and the results are as follows.

The anxiety about breathing difficulties during the exercise significantly decreased in the subjects who performed the six-minute walking exercise. Oh (2003) also reported that the psychological symptoms, such as insecurity and anxiety, showed significant improvement compared to the physical symptoms in patients with COPD after two weeks of respiratory rehabilitation, which is in agreement with the results of this study. The subjects of this study were elderly patients with pneumoconiosis and who were isolated from their family and from the society due to their long-term hospitalization. As such, the training and support from the nurses for the walking exercise seemed to help decrease their anxiety about breathing difficulties during the exercise. Also, the continuous walking exercise, three times a week for 10 weeks, was considered helpful in building up their confidence about exercise, which eventually led to a decrease in their anxiety about dyspnea. Oh (2003) reported that the physical symptoms experienced by most
COPD patients are dyspnea, fatigue, and sleep disturbance, and that their psychological symptoms included insecurity, lethargy/ despair, and anxiety. The previous studies (Kim and Kang, 2006) emphasized the importance of psychological intervention for relieving dyspnea. Leidy and Haase (1996) reported that COPD patients have anxiety about breathing difficulties when carrying out physical activities, which limits their daily activities. As such, reliving anxieties through respiratory rehabilitation programs such as an exercise program is considered very important as it affects the quality of life associated with ADL in patients with pneumoconiosis.

The MBS of the subjects who performed a six-minute exercise significantly decreased compared to that of the subjects who did not perform such exercise. This result is in agreement with the results of the previous studies (Han, 2003; Kim, 2001), which reported improved dyspnea after the application of an exercise program as a part of the respiratory rehabilitation program for COPD patients. American College of Chest Physicians/American Association of Cardiovascular and Pulmonary Rehabilitation (ACCP/ AACVPR, 1997) also reported that a respiratory rehabilitation program significantly improved dyspnea during exercise and daily activities, and that training with exercise helped more in maintaining the efficacy of decreased dyspnea. The previous studies used different tools, however, to measure the patients' dyspnea. As such, further studies using an assessment tool that includes comprehensive symptoms such as other respiratory symptoms apart from dyspnea, ADL, sleep, and confidence, such as the COPD assessment test (CAT) dose, are being considered to be performed in the future. 
The six-minute walking exercise did not affect the subjects' FEV1\% pred. and FEV1/FVC\% as such results did not show a significant difference. This result is in agreement with the results of the previous studies (Haave et al., 2008; Kongsgaard et al., 2004; Troosters et al., 2000) that investigated the change in the pulmonary function after aerobic and cardio exercises in COPD patients and reported no change in the FEV1\% pred. and FEV1/ FVC\% results. Covey et al. (2001) and Parker et al. (2005) reported that the pulmonary function is not a good indicator for dyspnea as no significant relationship between the dyspnea and pulmonary function results was found in their study. Moreover, Kim (2001) and Panton et al. (2004) reported degenerated FEV1/ FVC\% and FEV1\% pred. results after exercise. The poor results of their studies were attributed to the short study period (between 4 and 24 weeks) and to the non-consistent exercise types, including aerobic and upper/lower-limb cardio exercises, because it is difficult to recover the pulmonary function that has degenerated for a long time within a few weeks or months. Jang and Kim (2006) recommended consistent exercise as it can slow down the progression of pulmonary function degeneration and can bring about positive effects even if the exercise cannot bring the function back to normal.

The subjects who performed the six-minute walking exercise showed significant improvements in ADL compared to those who did not perform the exercise. This result is in agreement with that of the study conducted by Han (2003), which reported improved ADL after the implementation of the respiratory rehabilitation program, and with the study conducted by Chen et al. (2011), which reported a higher activities-of-daily-living score after one year of exercise in serious COPD patients. As such, exercise was considered effective for improving ADL. Lareauet et al. (1996) reported that decreased ADL is associated with dyspnea or fatigue in COPD patients, and suggested that the use of an intervention for dyspnea can effectively improve ADL. The study conducted by Satoh et al. (2009) on ADL based on the subject's symptoms reported that the patients who experienced severer dyspnea showed more decreased ADL, which explains the correlation between dyspnea and ADL. This study also showed that walking exercise decreased the index of dyspnea and increased ADL, which proved that walking exercise can be easily performed anytime during the conduct of daily activities by pneumoconiosis patients. Also, it was confirmed that the six-minute walking test has a significant meaning as a test method for nursing and exercise intervention in chronic pulmonary disease patients.

As such, this study confirmed that the six-minute walking ex- ercise can decrease the anxiety about breathing difficulties during the exercise, can lower the dyspnea index after the exercise, and can improve ADL in pneumoconiosis patients. The six-minute walking exercise is expected to be used as a nursing intervention for the respiratory rehabilitation of pneumoconiosis patients.

This study was conducted to investigate the effect of the sixminute walking exercise through the six-minute walking test on subjective dyspnea and ADL in pneumoconiosis patients aged 60 years or more, and to provide basic data for nursing intervention for such patients. The study results showed that the six-minute walking exercise decreased the pneumoconiosis patient subjects' anxiety about breathing difficulties during the exercise and lowered the dyspnea index after the exercise, which eventually improved ADL in the subjects. As such, this six-minute walking exercise was considered an easily applicable exercise with a minimum cost for respiratory rehabilitation, and was regarded as capable of reducing the burden of exercise on the patients as it involves a similar amount of exercise as the daily activities, thus motivating the patients and improving their confidence about exercise. The six-minute walking exercise is expected to be used as a nursing intervention for the respiratory rehabilitation of pneumoconiosis patients. Moreover, more exercise programs should be constantly developed for respiratory rehabilitation.

\section{CONFLICT OF INTEREST}

No potential conflict of interest relevant to this article was reported.

\section{ACKNOWLEDGEMENTS}

The authors appreciate the willing participation of the pneumoconiosis patient subjects in this study, the support from their family, and the help extended by the medical staff and nurses of the $\mathrm{G}$ and $\mathrm{W}$ hospitals for data collection.

\section{REFERENCES}

ACCP/AACVPR pulmonary Rehabilitation Guidelines Pannel. Pulmonary rehabilitation joint AACP/AACVPR evidence-based guidelines. Chest 1997;112:1363-1396.

American Thoracic Society. ATS statement: guidelines for the six-minute walk test. Am J Respir Crit Care Med 2002;166:111-117.

American Thoracic Society. Standards for the diagnosis and care of pa- 
tients with chronic obstructive pulmonary disease. Am J Respir Crit Care Med 1995;152(12):77-124.

Bauer TTI, Heyer CM, Duchna HW, Andreas K, Weber A, Schmidt EW, Ammenwerth W, Schultze-Werninghaus G. Radiological findings, pulmonary function and dyspnea in underground coal mines. Respiration 2007;74:80-87.

Borg GA. Psychophysical bases of perceived exertion. Med Sci Sports Exerc 1982;14:377-381.

Chen GI, Zhou X, Hu X, Liu Y, Li Q. Effect of exercise on the quality of life and pulmonary function in patients with chronic obstructive pulmonary disease. J Cent South Univ (Med Sci) 2011;36: 682-686.

Covey MKI, Larson JL, Wirtz SE, Berry JK, Pogue NJ, Alex, CG, Patel, M (2001). High-intensity inspiratory muscle training in patients with chronic obstructive pulmonary disease and severely reduced function. J Cardiopulm Rehabil 2001;21:231-240.

Faul F, Erdfelder E, Lang AG, Buchner A. G*Power 3: a flexible statistical power analysis program for the social, behavioral, and biomedical sciences. Behav Res Methods 2007;39:175-191.

Guyatt, GHI, Berman LB, Townsend M, Pugsley SO, Chambers LW. A measure of quality of life for clinical trials in chronic lung disease. Thorax 1987;42:773-778.

Haave EI, Skumlien S, Hyland ME. Gender considerations in pulmonary rehabilitation. J Cardiopulm Rehabil Prev 2008;28(3):215-219.

Han SJ. The effects of a pulmonary rehabilitation program for chronic obstructive pulmonary disease patients. J Korean Acad Nurs 2003;33: 1008-1017.

Jang HJ, Kim JY. The effects of self-efficacy promoting pulmonary rehabilitation program in out-patients with chronic obstructive pulmonary disease. Tuberc Respir Dis 2006;61:533-546.

Kim AK. The study on the effects of a respiratory rehabilitation program for COPD patients. J Korean Acad Nurs 2001;31(2):257-267.

Kim HS, Kang HS. Effects of a pulmonary rehabilitation program for patients with chronic obstructive pulmonary disease. J Korean Clin Nurs Res 2006;12:43-53.

Kim US. Cardiopulmonary exercise testing: application to pulmonary rehabilitation. Tuberc Respir Dis 2003;55:135-139.

Korean Occupational Safety and Health Agency (2011). Industrial accident analysis. 69.
Kongsgaard MI, Backer V, J ørgensen K, Kjaer M, Beyer N. Heavy resistance training increases muscle size, strength and physical function in elderly male COPD patients-a pilot study. Respir Med 2004;98:10001007.

Lareau SCI, Breslin EH, Meek PM. Functional status instruments: outcome measure in the evaluation of patients with chronic obstructive pulmonary disease. Heart Lung 1996;25:212-224.

Lee HR, Kim SG, Jang J, Kim SG, Lee WY. A study on clinical applicability of dyspnea index and the 6-minute walking test in patients complaining of dyspnea. Korean J Intern Med 1992;42:790-801.

Leidy NK, Haase JE. Functional performance in people with chronic obstructive pulmonary disease: A qualitative analysis. ANS Adv Nurs Sci 1996;18(3):77-89.

No SR, Lee HE, Yoon GW, Lee EH, Kim JH, Oh AE, Baek, DM. Pneumoconiosis diagnosis method by checking the availability of the 6 minute walk. Korean J Occup Med 2009;43:647-648.

Oh HS. Meta-analysis on the effectiveness of pulmonary rehabilitation program on exercise capacity/tolerance and general health status. J Korean Acad Nurs 2003;33:743-752.

Parker CMI, Voduc N, Aaron SD, Webb KA, O'Donnell DE. Physiological changes during symptom recovery from moderate exacerbations of COPD. Eur Respir J 2005;26(3):420-428.

Panton LBI, Golden J, Broeder CE, Browder KD, Cestaro-Seifer DJ, Seifer FD. The effects of resistance training on functional outcomes in patients with chronic obstructive pulmonary disease. Eur J Appl Physiol, 2004;91:443-449.

Satoh HI, Iwashima A, Endo Y, Nakayama H, Hasegawa T, Suzuki E. Effect of proactive use of inhaled procaterol on dyspnea in daily activities and quality of life in patients with chronic obstructive pulmonary disease. Nihon Kokyuki Gakkai Zasshi 2009;47:772-780.

Sirajuddin AI, Kanne JP. Occupational lung disease. J Thorac Imaging 2009;24:310-320

Stark RD, Gambles SA, Chatterjee SS. An exercise test to assess clinical dyspnea: estimation of reproducibility and sensitivity. Br J Dis Chest 1982;76:269-278.

Troosters TI, Gooselink R, Decramer M. Short-and long-term effects of outpatient rehabilitation in patients with chronic obstructive pulmonary disease: a randomized trial. Am J Med 2000;109:207-212. 